\title{
Fine-scale Structures of Flux Ropes Tracked by Erupting Material
}

\author{
Ting $\operatorname{Li}^{1} \&$ Jun Zhang ${ }^{1}$
}

\begin{abstract}
We present the Solar Dynamics Observatory observations of two flux ropes respectively tracked out by material from a surge and a failed filament eruption on 2012 July 29 and August 04. For the first event, the interaction between the erupting surge and a loop-shaped filament in the east seems to "peel off" the filament and add bright mass into the flux rope body. The second event is associated with a C-class flare that occurs several minutes before the filament activation. The two flux ropes are respectively composed of $85 \pm 12$ and $102 \pm 15$ fine-scale structures, with an average width of about $1^{\prime \prime} .6$. Our observations show that two extreme ends of the flux rope are rooted in the opposite polarity fields and each end is composed of multiple footpoints (FPs) of the fine-scale structures. The FPs of the fine-scale structures are located at network magnetic fields, with magnetic fluxes from $5.6 \times 10^{18} \mathrm{Mx}$ to $8.6 \times 10^{19} \mathrm{Mx}$. Moreover, almost half of the FPs show converging motion of smaller magnetic structures over $10 \mathrm{hr}$ before the appearance of the flux rope. By calculating the magnetic fields of the FPs, we deduce that the two flux ropes occupy at least $4.3 \times 10^{20} \mathrm{Mx}$ and $7.6 \times 10^{20} \mathrm{Mx}$ magnetic fluxes, respectively.
\end{abstract}

Subject headings: Sun: corona — Sun: filaments, prominences — Sun: coronal mass ejections (CMEs)

\section{Introduction}

The flux rope is thought to be closely related to the coronal mass ejection (CME), which generally has a three-part structure: the bright core, the dark cavity and the leading edge (see e.g., Illing \& Hundhausen 1986). It is often believed that the twisted flux rope is the dark cavity which accumulates magnetic energy and mass within it (Chen 1996; Hudson \& Schwenn 2000; Gibson et al. 2006). The ideal magnetohydrodynamic (MHD) instabilities

\footnotetext{
${ }^{1}$ Key Laboratory of Solar Activity, National Astronomical Observatories, Chinese Academy of Sciences, Beijing 100012, China; [liting;zjun]@nao.cas.cn
} 
of the flux rope such as the kink instability and the torus instability are thought to be one type of mechanism that triggers the CME and associated activities (Török \& Kliem 2003, 2005; Fan 2005; Kliem \& Török 2006; Olmedo \& Zhang 2010). Thus, a detailed study of the flux rope is important for a clear understanding of CMEs, and this leads to a good ability to forecast CMEs and associated space weather.

The flux rope has been reconstructed in a complex magnetic topology from observed vector magnetograms by using non linear force-free field models (Guo et al. 2010; Canou \& Amari 2010; Jing et al. 2010). Moreover, the formation and dynamic behavior of flux ropes have been simulated successfully by many authors (Amari \& Luciani 1999; Amari et al. 2010; Fan \& Gibson 2004). In the simulation of Aulanier et al. (2010), the flux rope is progressively formed by flux-cancellation-driven photospheric reconnection in a bald-patch separatrix.

Recently, the direct observations of flux ropes during the eruption process have been reported by using the data from the Atmospheric Imaging Assembly (AIA; Lemen et al. 2012) onboard the Solar Dynamics Observatory (SDO; Pesnell et al. 2012). Cheng et al. (2011) presented the observation of a flux rope as a bright blob of hot plasma in the channel of $131 \AA$. The hot flux rope rapidly moved outward and stretched the surrounding magnetic field upward, similar to the classical magnetic reconnection scenario in eruptive flares. Detailed thermal property of the flux rope based on differential emission measure was investigated by Cheng et al. (2012). However, there is to our knowledge no observations of fine-scale structures of flux ropes tracked by erupting material. In this letter, we present two events of flux ropes and investigate their fine-scale structures and magnetic properties by using the data from SDO/AIA and the Helioseismic and Magnetic Imager (HMI; Schou \& Larson 2011).

\section{Observations and Data Analysis}

On 2012 July 29 and August 04, SDO/AIA observed two flux ropes that are respectively tracked by material from a surge and a failed filament eruption. Both of them are composed of thread-like structures, which warp and interweave together.

The $S D O /$ AIA takes full-disk images in 10 (E)UV channels at $1^{\prime \prime} .5$ resolution and high cadence of $12 \mathrm{~s}$. The flux ropes could be observed in all the 7 EUV channels. The 171 channel best shows the flux rope and we focus on this channel in this study. We also present the observations of flux ropes in different channels such as 304, 193, 335 and $131 \AA$.

The 5 EUV channels correspond to different temperatures: $171 \AA$ (Fe IX) at $0.6 \mathrm{MK}$, 
$304 \AA$ (He II) at $0.05 \mathrm{MK}, 193 \AA$ (Fe XII) at $1.5 \mathrm{MK}$ (with a hot contribution of Fe XXIV at $20 \mathrm{MK}$ and cooler $\mathrm{O} \mathrm{V}$ at $0.2 \mathrm{MK}$ ), $335 \AA$ (Fe XVI) at $2.5 \mathrm{MK}$ and $131 \AA$ (Fe VIII, Fe XXI) at 11 MK (O'Dwyer et al. 2010; Boerner et al. 2012; Parenti et al. 2012). We also use the full-disk line-of-sight magnetic field data from the HMI onboard $S D O$, with a cadence of $\sim 45 \mathrm{~s}$ and a sampling of $0^{\prime \prime} .5$ pixel $^{-1}$.

\section{Results}

\subsection{Overview of the Two Flux Ropes}

At about 00:30 UT on 2012 July 29, a surge occurred in NOAA AR 11530 (S19W00) and plenty of material was ejected northward (see Figure $1 b$ and Animation 1 in the online journal). By examining the SDO/HMI line-of-sight magnetograms, we found that the magnetic flux cancellation took place several hours prior to the surge (Figure $1 d$ ). The obvious brightening was observed at $171 \AA$ at the location of the cancelled flux. This cancellation could be what led to the occurrence of the surge. When the surge first appears and starts to ascend, there is evidence of interaction between it and a loop-shaped filament in the east side of the erupting surge (see Figure $1 e$ and Animation 2 in the online journal). This interaction seems to "peel off" the filament and to add mass into the flux rope body. Simultaneously, brightenings at the interaction location and the footpoint of the surge at $304 \AA$ are also observed (see Figure $1 e$ and Animation 2 in the online journal).

At about 00:52 UT, the erupting material seemed to be confined and moved toward the west (see Animation 1 in the online journal). Meanwhile, the bright fine-scale structures are clearly observed. About 52 min later (01:44 UT), the erupting material arrived at the west end of the flux rope and the entire flux rope was tracked out by the ejected material (see Figure 1c). It seems that the surge occurs within the flux rope and the footpoint of the surge is located at one end of the flux rope, and thus the material from the surge flows along the flux rope. The approximate length of the flux rope is $596 \mathrm{Mm}$, and the apparent flow speed of the material along the flux rope body is about $150 \mathrm{~km} \mathrm{~s}^{-1}$. The flux ropes observed at 304, 193, 335 and $131 \AA$ are roughly the same on the whole. However, they are different in some details. Taking the area denoted by white rectangles in Figure 1 for example, the 193 $\AA$ observations are similar to those of $171 \AA$, and the 304, 335 and $131 \AA$ observations are different from $171 \AA$ observations as the fine-scale structures (pointed by white arrows) are not clearly identified in these three channels.

On 2012 August 04, the second flux rope was observed in NOAA AR 11539 (S23E32). Before the flux rope was tracked, there existed a filament at the east part of the flux rope 
(see Figure 2a). At 11:04 UT, a C2.9 flare occurred at the east of the filament (see Figure $2 e$ and Animation 4 in the online journal), which peaked at 11:47 UT and ended at 12:49 UT. At about 11:14 UT, the filament started to turn over and brighten (Figure 2b). At about 11:40 UT, the ejected material from the filament successively moved toward the southwest (see Animation 3 in the online journal). Then the arch-shaped flux rope with helical finescale structures was observed clearly (Figure $2 c$ ). The observed length of the flux rope is about $546 \mathrm{Mm}$, and it takes the filament material about $51 \mathrm{~min}$ to flow from the east to the west end. The apparent flow velocity along the flux rope body is approximately $180 \mathrm{~km}$ $\mathrm{s}^{-1}$. Similar to the first flux rope on 2012 July 29, the fine-scale structures denoted by the white arrow in white rectangles of Figure 2 are identified clearly at 171 and $193 \AA$ and seems obscure at 304, 335 and $131 \AA$. At about 16:00 UT on August 6, the east part of the second flux rope erupted, and this eruption resulted in a faint CME with a speed of about $260 \mathrm{~km}$ $\mathrm{s}^{-1}$. The whole flux rope erupted at about 03:00 UT on August 8, associated with a halo CME with a speed of about $230 \mathrm{~km} \mathrm{~s}^{-1}$.

\subsection{Fine-scale Structures of the Two Flux Ropes}

As the erupting material arrived at the west extreme end of the first flux rope on 2012 July 29, the west end showed obvious brightening (Figures $1 c$ and $3 a$ ). Then partial material went back toward the east and brightened the east end at about 03:02 UT (see Figure $3 d$ and Animation 1 in the online journal). The brightening at the ends makes it possible to determine the ends location. Thus we select two areas (denoted by red and blue rectangles in Figure 1c) to investigate the ends and fine-scale structures.

By counting the number of fine-scale structures one by one, we notice that the first flux rope is composed of $85 \pm 12$ fine-scale structures, and 15 well identified fine-scale structures are selected to measure their widths. Two examples are shown in Figures $3 c, f$ and $g$. Firstly, the intensity-location profiles (black curves in Figures $3 f$ and $g$ ) along slices perpendicular to the fine-scale structures (Slices "S1" and "S2" in Figure 3c) are obtained. Secondly, we use Gaussian function to fit the intensity-location profiles and two Gaussian fitting profiles (blue and red ones) are shown in Figures $3 f$ and $g$. The full width at half maximum (FWHW) of the Gaussian fitting profile is thought to be the width of fine-scale structure. The average width of these fine-scale structures is $1^{\prime \prime} .8$, with the maximum value of $2^{\prime \prime} .0$ and the minimum value of $1^{\prime \prime} .4$.

For the first flux rope, there are 12 western footpoints (FPs) of the fine-scale structures that form the west end of the flux rope (white circles in Figure 3a). By comparing the 171 $\AA$ observations with line-of-sight magnetograms, we find that all the western FPs are rooted 
in negative polarity fields (Figure $3 b$ ). The net magnetic fluxes of these FPs are in the range of $8.6 \times 10^{18}-8.6 \times 10^{19} \mathrm{Mx}$. The magnetic flux of the west end of the flux rope is $-4.3 \times 10^{20}$ $\mathrm{Mx}$. This is the lower limit of the magnetic flux since some FPs are not identified and calculated for they are not accompanied by brightening. The 8 eastern FPs are rooted in positive polarity fields (Figures $3 d$ and $e$ ). The net magnetic fluxes of eastern FPs are from $5.6 \times 10^{18}$ to $2.8 \times 10^{19} \mathrm{Mx}$. The magnetic flux of the east end of the flux rope is $1.3 \times 10^{20}$ $\mathrm{Mx}$, which is less than that of the western end. Not all the eastern FPs of the fine-scale structures are identified and thus there exists the discrepancy between the two sides.

For the second flux rope on 2012 August 04, we similarly select two areas where the brightening occurs at the ends (Figures $2 c$ and 4 ). This arch-shaped flux rope seems more complex than the first one and has two main western ends (one in Figure $4 a$ and the other one in Figure $4 d$ ) which are separated apart. The flux rope is composed of $102 \pm 15$ finescale structures. The average width of 20 clearly identified fine-scale structures is $1^{\prime \prime} .5$. The thickest one has a width of $1^{\prime \prime} .7$, and the thinnest one has that of $1^{\prime \prime} .1$ (Figures $4 c, f$ and $g$ ). The line-of-sight magnetograms show that 22 western FPs (15 ones in Figures $4 a-b$ and 7 ones in Figures $4 d-e$ ) of the fine-scale structures are anchored at positive polarity fields (Figures $4 a-b, d-e$ ). The net magnetic fluxes of these western FPs are in the range of $1.1-8.1 \times 10^{19} \mathrm{Mx}$. The total magnetic flux of western ends of the flux rope is $7.6 \times 10^{20} \mathrm{Mx}$. The eastern end of the flux rope is close to the solar limb and not accompanied by EUV enhancements, thus it could not be identified.

By examining the magnetic field evolution at the FPs of the fine-scale structures over 10 hr before the appearance of the flux rope, we find almost half of these FPs show converging motion of smaller magnetic structures for both the flux ropes. Figure 5 presents one example of the eastern FPs of the first flux rope. As seen in the stack plot along Slice "A-B", the west magnetic structure obviously moved toward the east one with a velocity of $0.2 \mathrm{~km} \mathrm{~s}^{-1}$ and the motion of the east one was slower than the west one, with a velocity of $0.1 \mathrm{~km} \mathrm{~s}^{-1}$ (Figure 5e).

\section{Summary and Discussion}

We present the SDO/AIA observations of two flux ropes on 2012 July 29 and August 04 which are tracked out by material from a surge and a failed filament eruption. For the two flux ropes, the apparent speeds of filling of the flux rope structure with chromospheric and coronal plasma are respectively 150 and $180 \mathrm{~km} \mathrm{~s}^{-1}$, which are comparable to the typical sound speed for the corona of about $100-200 \mathrm{~km} \mathrm{~s}^{-1}$. For the first flux rope, the approximate length of the flux rope is $596 \mathrm{Mm}$. By examining the fine-scale structures which are observed 
more clearly, we roughly estimate the twist is about $\pi$. For event 2 , the observed length of the flux rope is about $546 \mathrm{Mm}$, and the average twist is about $2 \pi$. Seen from the SolarTerrestrial Relations Observatory (STEREO; Kaiser et al. 2008) B viewpoint, the two flux ropes are both located at the west limb. By using three-dimensional reconstructions, we obtain the heights of the two flux ropes, which are respectively 90 and $140 \mathrm{Mm}$ above the solar surface. The two flux ropes analyzed here are respectively composed of $85 \pm 12$ and $102 \pm 15$ fine-scale structures, which probably outline the magnetic field structures of flux ropes (Martin et al. 2008; Lin 2011). The width of the fine-scale structures ranges from $1^{\prime \prime} .1$ to $2^{\prime \prime} .0$, with an average of about $1^{\prime \prime} .6$. It is comparable to the resolution limit of the AIA telescope of about $1^{\prime \prime} .2$, which suggests that even thinner structures may exist. Moreover, the width of the fine-scale structures is an order of magnitude larger than the ultrafine magnetic loop structures observed by Ji et al. (2012).

Before the flux ropes are tracked out by erupting material, part of magnetic flux rope structures may exist in the space filled in by the flux ropes. For event 1, there are several similar events in two days before the appearance of the first flux rope on 2012 July 29. Moreover, the $304 \AA$ images shortly before the surge injection and flux rope appearance reveal the presence of long and thin absorbing threads along the first flux rope. For event 2, the filament at the east location of the flux rope may indicate part of the pre-existing flux rope structures.

When the surge in event 1 appears and the filament in event 2 is activated, the obvious brightenings and flare activities are observed simultaneously at the east of the two flux ropes. This implies that heating takes place and may illuminate the flux rope body by filling it with hot and dense plasma emitting in the EUV channels. This is similar to the observations of Raouafi (2009), who suggested that a C-class flare near one footpoint of the flux rope led to the brightening of the magnetic structure showing its fine structure. While the material arrives at the FPs of the fine-scale structures, the FPs are consequently brightened. The brightening at the FPs may be caused by the conversion from the kinetic energy to the thermal energy.

Our observations show that there exist $7-15$ FPs of the fine-scale structures for each end of the flux rope. By comparing the EUV observations with the HMI magnetograms, we find that the FPs at one end of the flux rope on July 29 are rooted in the same polarity fields and the FPs at the other end are anchored at the opposite polarity fields (Figure 3). For the flux rope on August 04, the eastern end could not be identified and only the western end is analyzed here. The FPs of the fine-scale structures are located at network magnetic fields and their magnetic fluxes are in the range of $5.6 \times 10^{18}-8.6 \times 10^{19} \mathrm{Mx}$. The magnetic fluxes of the two flux ropes are at least $4.3 \times 10^{20}$ and $7.6 \times 10^{20} \mathrm{Mx}$. According to the statistical study 
of Sung et al. (2009), the magnetic flux of 34 magnetic clouds (MC) for in-situ observations varies from $1.25 \times 10^{18}$ to $4.69 \times 10^{21} \mathrm{Mx}$ with the average of $1.1 \times 10^{21} \mathrm{Mx}$. The magnetic flux of the flux ropes in our observations is comparable to that of the MC. Moreover, almost half of the FPs of the fine-scale structures show converging motion of smaller magnetic structures over $10 \mathrm{hr}$ before the appearance of the flux rope. The network magnetic field is often thought to be the converging center (Zhang et al. 1998), which is consistent with our observations. As small-scale magnetic fields located at the converging centers always exist tens of hours (Liu et al. 1994), implying that the flux ropes have a relatively long lifetime.

The flux ropes are observed in all the 7 EUV channels (304, 171, 193, 211, 335, 94 and $131 \AA$ ) of the SDO/AIA that cover the temperature from 0.05 MK to $11 \mathrm{MK}$. This is consistent with recent observations of Patsourakos et al. (2013) and Li \& Zhang (2013), who reported the hot and cool components of flux ropes. However, there exist the flux ropes that

could only be observed in hot channels such as 94 and $131 \AA$ (Zhang et al. 2012; Cheng et al. 2011, 2012). The comprehensive characteristics of flux ropes need to be analyzed in further studies.

We acknowledge the SDO/AIA and HMI for providing data. This work is supported by the National Basic Research Program of China under grant 2011CB811403, the National Natural Science Foundations of China (11025315, 11221063, 10921303 and 11003026), the CAS Project KJCX2-EW-T07, and the Young Researcher Grant of National Astronomical Observatories, Chinese Academy of Sciences.

\section{REFERENCES}

Amari, T., Aly, J.-J., Mikic, Z., \& Linker, J. 2010, ApJ, 717, L26

Amari, T., \& Luciani, J. F. 1999, ApJ, 515, L81

Aulanier, G., Török, T., Démoulin, P., \& DeLuca, E. E. 2010, ApJ, 708, 314

Boerner, P., Edwards, C., Lemen, J., et al. 2012, Sol. Phys., 275, 41

Canou, A., \& Amari, T. 2010, ApJ, 715, 1566

Chen, J. 1996, J. Geophys. Res., 101, 27499

Cheng, X., Zhang, J., Liu, Y., \& Ding, M. D. 2011, ApJ, 732, L25

Cheng, X., Zhang, J., Saar, S. H., \& Ding, M. D. 2012, ApJ, 761, 62 
Fan, Y. 2005, ApJ, 630, 543

Fan, Y., \& Gibson, S. E. 2004, ApJ, 609, 1123

Gibson, S. E., Foster, D., Burkepile, J., de Toma, G., \& Stanger, A. 2006, ApJ, 641, 590

Guo, Y., Schmieder, B., Démoulin, P., et al. 2010, ApJ, 714, 343

Hudson, H., \& Schwenn, R. 2000, Advances in Space Research, 25, 1859

Illing, R. M. E., \& Hundhausen, A. J. 1986, J. Geophys. Res., 91, 1095

Ji, H., Cao, W., \& Goode, P. R. 2012, ApJ, 750, L25

Jing, J., Yuan, Y., Wiegelmann, T., et al. 2010, ApJ, 719, L56

Kaiser, M. L., Kucera, T. A., Davila, J. M., St. Cyr, O. C., Guhathakurta, M., \& Christian, E. 2008, Space Sci. Rev., 136, 5

Kliem, B., \& Török, T. 2006, Physical Review Letters, 96, 255002

Li, L. \& Zhang, J. 2013, A\&A, 552, L11

Lin, Y. 2011, Space Sci. Rev., 158, 237

Liu, Y., Zhang, H., Ai, G., Wang, H., \& Zirin, H. 1994, A\&A, 283, 215

Lemen, J. R., Title, A. M., Akin, D. J., et al. 2012, Sol. Phys., 275, 17

Martin, S. F., Lin, Y., \& Engvold, O. 2008, Sol. Phys., 250, 31

O’Dwyer, B., Del Zanna, G., Mason, H. E., Weber, M. A., \& Tripathi, D. 2010, A\&A, 521, A21

Olmedo, O., \& Zhang, J. 2010, ApJ, 718, 433

Patsourakos, S., Vourlidas, A., \& Stenborg, G. 2013, ApJ, 764, 125

Parenti, S., Schmieder, B., Heinzel, P., \& Golub, L. 2012, ApJ, 754, 66

Pesnell, W. D., Thompson, B. J., \& Chamberlin, P. C. 2012, Sol. Phys., 275, 3

Raouafi, N.-E. 2009, ApJ, 691, L128

Schou, J., \& Larson, T. P. 2011, Bulletin of the American Astronomical Society, 1605

Sung, S.-K., Marubashi, K., Cho, K.-S., et al. 2009, ApJ, 699, 298 
Török, T., \& Kliem, B. 2003, A\&A, 406, 1043

Török, T., \& Kliem, B. 2005, ApJ, 630, L97

Zhang, J., Cheng, X., \& Ding, M.-D. 2012, Nature Communications, 3, 747

Zhang, J., Wang, J., Wang, H., \& Zirin, H. 1998, A\&A, 335, 341

This preprint was prepared with the AAS IATEX macros v5.2. 


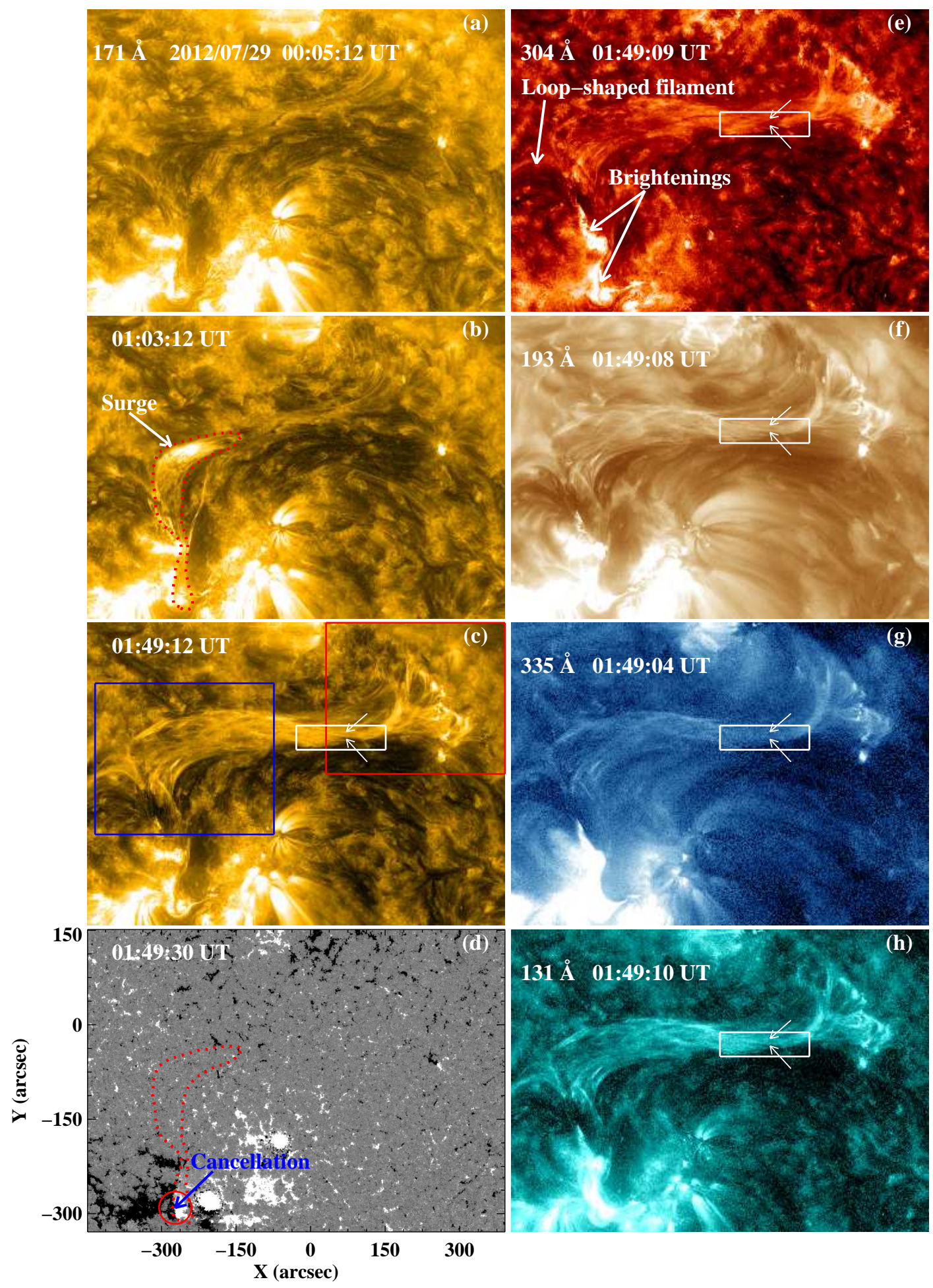

Fig. 1. - SDO/AIA multi-wavelength images and HMI line-of-sight magnetogram showing the evolution of the flux rope on 2012 July 29 (see Animations 1 and 2, available in the online edition of the journal). The red solid rectangle in panel $c$ denotes the FOV of Figure $3 a$ and the blue one denotes the FOV of Figure $3 d$. 


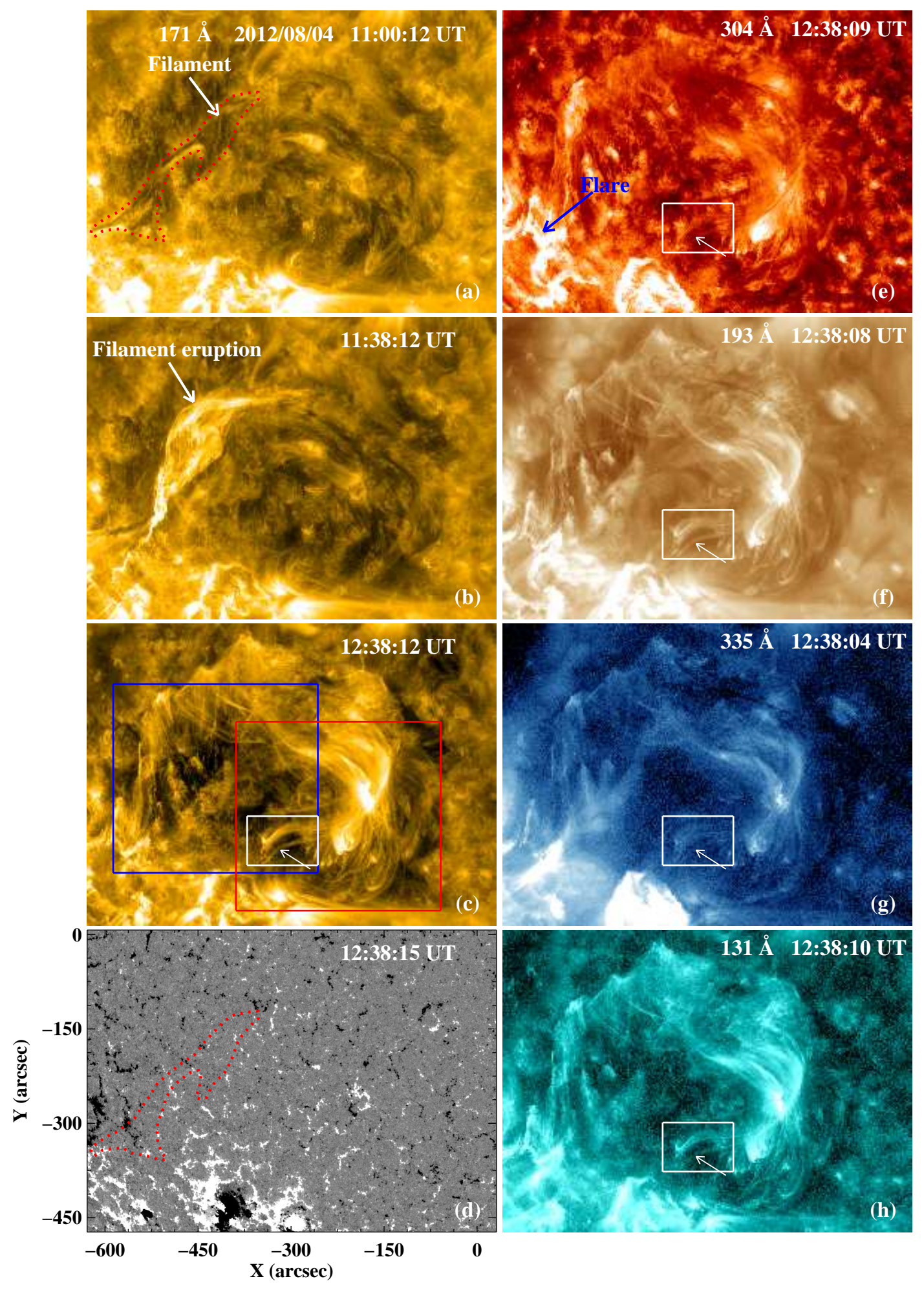

Fig. 2.- SDO/AIA multi-wavelength images and HMI line-of-sight magnetogram showing the evolution of the flux rope on 2012 August 04 (see Animations 3 and 4, available in the online edition of the journal). The red solid rectangle in panel $c$ denotes the FOV of Figure $4 a$ and the blue one denotes the FOV of Figure $4 d$. 

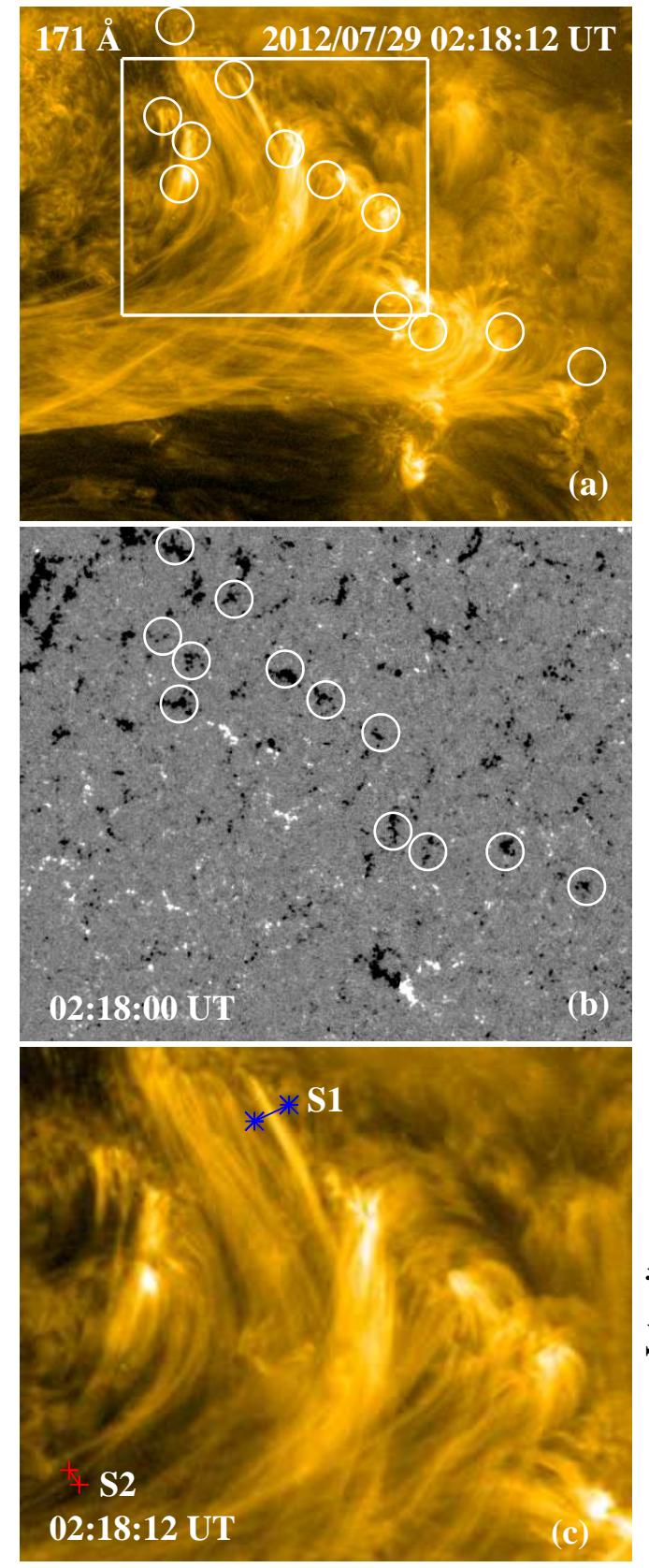

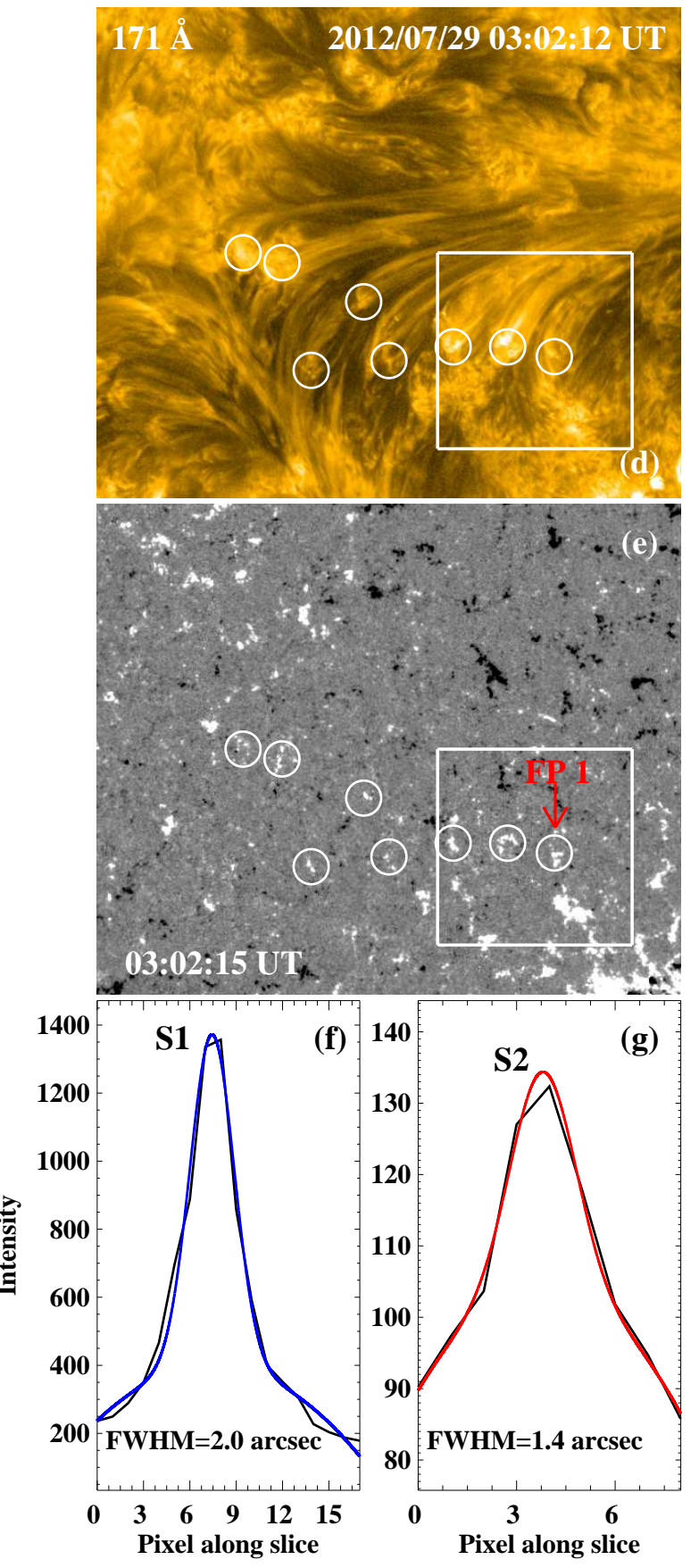

Fig. 3.- SDO/AIA $171 \AA$ images and HMI magnetograms showing the western (panels $a$ and $b$ ) and eastern ends (panels $d$ and $e$ ) of the flux rope on 2012 July 29, and the Gaussian fitting profiles (panels $f$ and $g$ ) showing the widths of fine-scale structures. The white rectangle in panel $a$ denotes the FOV of panel $c$ and white rectangles in panels $d$ and $e$ denote the FOV of Figure 5. The blue and red curves in panels $f$ and $g$ are respectively the Gaussian fitting profiles of the intensity-location curves (black ones) along the blue and red slices (Slices "S1" and "S2") in panel $c$. 

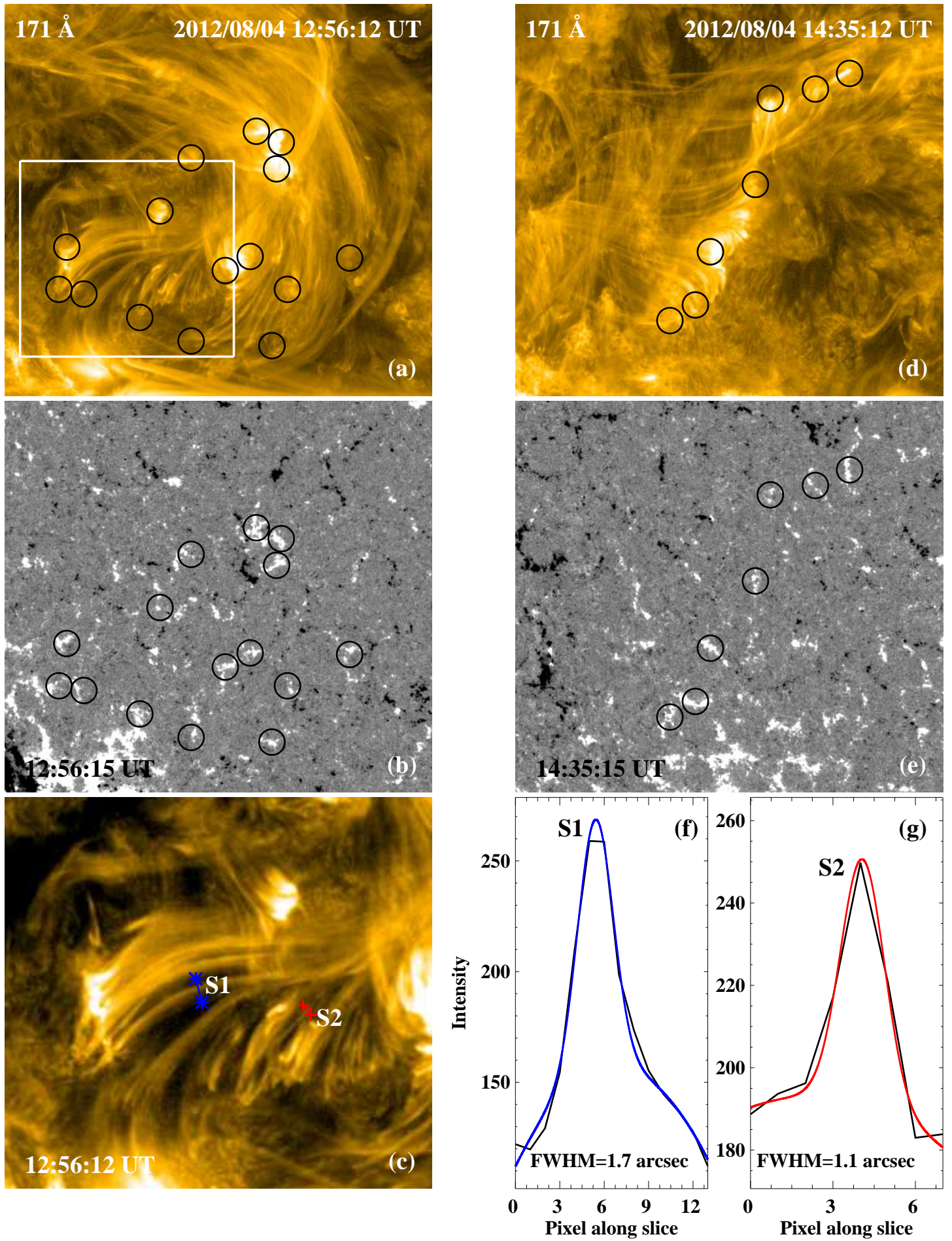

Fig. 4.- SDO/AIA $171 \AA$ images and HMI magnetograms showing two main western ends of the flux rope (one in panels $a-b$ and the other in panels $d-e$ ) on 2012 August 4, and the Gaussian fitting profiles (panels $f$ and $g$ ) showing the widths of fine-scale structures. The white rectangle in panel $a$ denotes the FOV of panel $c$. 

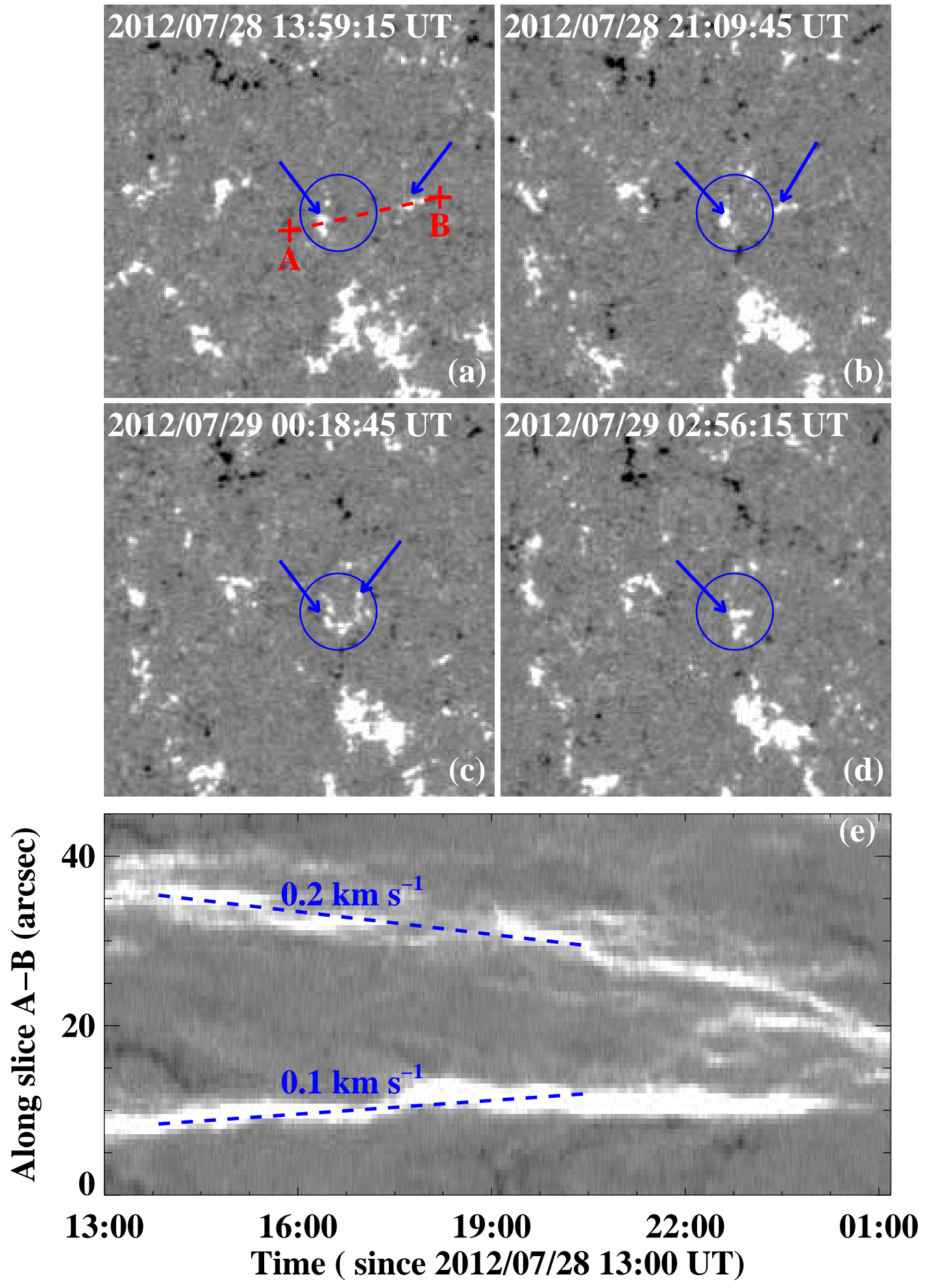

Fig. 5.- SDO/HMI magnetograms showing the converging motion of smaller magnetic structures at the FPs of the fine-scale structures. Blue circles denote one of the eastern FPs ("FP 1" in Figure 3e) of the fine-scale structures for the first flux rope on 2012 July 29. The stack plot along Slice "A-B" (red dashed line in panel $a$ ) is shown in panel $e$. 